\title{
Communication and conditioning: Correlated reinforcement
}

\author{
ROBERT FRANK WEISS and MICHAEL J. CLUTS \\ University of Oklahoma, Norman, Oklahoma 73019 \\ MARY JANE WILLIAMS \\ Department of Marketing and General Business, \\ Murray State University, Murray, Kentucky 42071
}

and

\author{
FRANKLIN G. MILLER \\ Department of Consumer Sciences, Purdue University, West Lafayette, Indiana 47907
}

\begin{abstract}
This is the third in a series of studies concerned with various forms of correlated reinforcement (discrete-trials DRL) in communication. Speaking in reply functions as a reinforcer. In an experimental conversation modeled on discrete-trials instrumental conditioning, with speed as the dependent variable, subjects learned an instrumental response reinforced by the opportunity to speak in reply. When shorter delay of the opportunity to reply was made contingent upon subjects' responding slowly, subjects learned to match the cut-off speed, while noncorrelated yoked controls showed an ordinary acquisition curve, as in conditioning studies of correlated delay of reinforcement.
\end{abstract}

Latency data from a continuing series of experiments in interpersonal communication show a pattern of results which bears a striking functional similarity to discrete-trials instrumental conditioning. In these studies speaking in reply has been found to function as a reinforcer, and subjects will learn an instrumental response the reinforcement for which is the opportunity to speak in reply to a person of differing opinion. Experimental demonstration of our theoretical analogies extends well beyond the analogs of acquisition and extinction, commonly deemed sufficient in social psychology or behavior therapy, to include analogs of partial reinforcement effects, intermittent shock effects, delay of reinforcement effects, drive effects, correlated reinforcement effects (discrete-trials DRL), and correlated delay of reinforcement effects (e.g., Weiss, Beck, \& Stich, 1972; Weiss, Boyer, Colwick, \& Moran, 1971). Exploration of such a broad range of predicted effects makes it particularly difficult to include demonstration of the replicability of these effects within the program of research, but we have, nevertheless, taken care to show these effects to be replicable and now do so with correlated delay of reinforcement.

In Logan's (1960) correlated reinforcement procedure, reinforcement can be made contingent upon the subject's responding with a longer latency than a certain

Reprints may be obtained from R. F. Weiss, Department of Psychology, University of Oklahoma, Norman, Oklahoma 73019. cut-off value. Under such a procedure, subjects learn to respond slowly, approximating the cut-off value. Studies of correlated reinforcement effects on response speed in human conditioning have typically been limited to the simplest condition, in which slow responses are reinforced and fast responses are not (Cairns \& Proctor, 1968; Weiss et al., 1971). In correlated delay studies, slow responses may be reinforced immediately and fast responses reinforced with delayed reinforcement. An appropriate delay value would have to be longer than the latency cut-off defining a slow response, so that fast responses would truly be penalized by effectively delayed reinforcement, while not being so long a delay as to amount to nonreinforcement. The necessary information was available from a 6-point delay of reinforcement gradient obtained in a previous study of speaking in reply (Weiss et al., 1971). Correlated reinforcement experiments typically involve a group of yoked controls, each one of which receives the same sequence of immediate and delayed reinforcements as his experimental partner but with these delays not contingent upon the control subject's speed. Such a control group is, in essence, an ordinary varied-delayed group and shows an ordinary learning curve, while the correlated delay group learns to respond slowly. If speaking in reply functions as a reinforcer, then the characteristic conditioning pattern of results should emerge for an instrumental response reinforced by the opportunity to speak in reply: ordinary acquisition in the yoked control group, compared with slow responding in the correlated delay of reply group. 


\section{METHOD}

On each trial, the subject listened to another person's viewpoint and could then press a switch (the instrumental response), the reinforcement for which was the opportunity to speak in reply to the other person.

\section{Deception and Masking Task}

The experiment was represented to the subjects as a study of opinion change. "We are interested in how your opinion may be affected by what someone else says, how your opinion may be affected by what you yourself say, and how what you say may affect the opinions of someone else." As a masking task, after each statement and reply, the subject indicated whether he had changed his opinion by moving a dial. Questionnaire data showed that subjects believed this deception; the switch appeared to them as a mere incidental to the serious business of opinion change through conversation. The "other person," simulated by a tape recorder, was said to be in another room from the subject, and the experimenter was in fact in another room. The subject addressed the "other person" and the experimenter over an intercom system and could also hear the experimenter give instructions and occasional comments ("Speak a little louder, Subject A") to the nonexistent other person.

\section{Apparatus and Procedure}

The subject was seated at a table facing the control room wall, which included four one-way vision windows. Instructional signals appeared in each window upon illumination. The signals were the large printed words (1) "listen," (2) "throw switch if you wish to comment," (3) "talk," and (4) "move dial to final opinion." A panel mounted on the table top contained the subject's "comment" switch, his intercom, and the maskingtask opinion-change dial.

An experimental trial began with the "listen" signal and the playing of the taped topic and opinion by the other person. When the taped message ended, the experimenter operated the control which simultaneously (1) presented the CS, the signal "throw switch if you wish to comment," and (2) started the latency timer. When the subject threw the comment switch, the latency timer automatically stopped, measuring latency to $.01 \mathrm{sec}$. The "talk" signal followed the switch-press response after a delay automatically regulated by an interval timer, and the subject spoke in reply. The procedure described was closely modeled on traditional discrete-trials instrumental conditioning. The reinforcement (speaking in reply) was contingent on the instrumental response (switch pressing). The dependent variable was speed (100/latency), measured from the time of the presentation of the CS (signal "press switch if you wish to comment") to the instrumental switch-pressing response.

The latency cut-off was $1.5 \mathrm{sec}$. In the correlated delay group, an interval timer turned on the "talk" signal immediately after the switch was pressed if latency was longer than $1.5 \mathrm{sec}$ and automatically delayed the "talk" signal $7.5 \mathrm{sec}$ if latency was shorter than $1.5 \mathrm{sec}$. For further details of delay procedure, see Weiss et al., 1971, 1972.

\section{Subjects and Materials}

The subjects were 80 undergraduates from the introductory psychology pool, 40 in each condition. The experiment was counterbalanced for sex, even though previous research indicated no main or interactive effects of sex.
The opinions expressed by the "other person" were selected by means of a questionnaire administered to 100 subjects from the introductory psychology pool. Selection criteria were that the opinions should be reasonably consistent with each other and dissimilar from the opinions of the introductory psychology students. The "other person" cassette tapes were the same sex as the subject, had a moderate regional accent characteristic of in-state students, were carefully gauged to approximate the articulateness and sophistication of a typical subject, and were presented in four randomized orders counterbalanced within each experimental condition.

\section{RESULTS AND DISCUSSION}

We found results in interpersonal communication which are analogous to correlated delay of reinforcement effects in instrumental conditioning: ordinary acquisition in the yoked control group compared with slow responding in the correlated delay of reply group. Initially, response speeds of both groups were slower than the cut-off speed. On the first of the 12 trials, the correlated group was actually somewhat faster than the controls; this difference reversed over the course of training so that the correlated group learned to perform more slowly than the controls, as predicted, with the correlated group closely matching the cutoff speed $(p<.05)$. Yoked controls received the same sequence of immediate and delayed reinforcement as the correlated group, so it is clear that the slow performance of the correlated group cannot be attributed to mere delay, and acquisition in the yoked controls cannot be attributed to mere practice; the difference in performance follows from the difference in reinforcement contingencies, noncorrelated controls vs correlated delay.

\section{REFERENCES}

CaIrns, R. B., \& Proctor, S. Selective reinforcement of response speeds in children. Journal of Experimental Psychology, 1968, 77, 168-170.

LogAN, F. A. Incentive. New Haven: Yale University Press, 1960.

Weiss, R. F., BECK, C. M., \& STICH, M. H. Correlated delay of reinforcement in the instrumental conditioning of conversational behavior. Psychonomic Science, 1972, 28, 211-212.

Weiss, R. F., Boyer, J. L., Colwick, J. T., \& Moran, D. J. A delay of reinforcement gradient and correlated reinforcement in the instrumental conditioning of conversational behavior. Journal of Experimental Psychology, 1971, 90, 33-38.

(Received for publication February 24, 1977.) 